\title{
Evaluating Passive Neighborhood Discovery for Low Power Listening MAC Protocols
}

\author{
Hamed Khanmirza \\ School of Electrical and \\ Computer Engineering \\ University of Tehran, Tehran, Iran \\ Email: hamed.khanmirza@ut.ac.ir \\ Telephone: +98 (21) 6111-4352
}

\author{
Olaf Landsiedel \\ Computer Science and Engineering \\ Chalmers University of Technology \\ Email: olafl@chalmers.se
}

\author{
Marina Papatriantafilou \\ Computer Science and Engineering \\ Chalmers University of Technology \\ Email: ptrianta@chalmers.se
}

\begin{abstract}
Low Power Listening (LPL) MAC protocols are widely used in today's sensors networks for duty cycling. Their simplicity and power efficiency ensures a long network life when nodes are battery driven and their easy deployment and lower cost of maintenance makes them suitable to be used in hard-toaccess places and harsh conditions. We argue that to fully utilize energy efficiency provided by LPL, other protocols in the protocol stack should be aware of mechanisms. In this paper, we focus on neighborhood discovery protocols and discuss their energy efficient integration with LPL. Then, we study the possibility of using a completely passive approach for neighborhood discovery in such networks and provide an analytical model for its performance characteristics. We verify our performance model both by simulation and implementation in TinyOS. Our evaluation results confirm the efficiency of our proposed method in dutycycled sensor networks.
\end{abstract}

Keywords-wireless sensor networks, energy efficient protocol design, neighborhood discovery, low power listening MAC protocols

\section{INTRODUCTION}

Neighborhood discovery is an essential building block for multi-hop wireless networks operation. With neighborhood discovery each node in the network tries to find and maintain a set of neighbors to communicate with other parts of the network that are not in that node's transmission range. One common and simple method to discover neighbors which is used in wired or wireless networks is broadcasting a periodic discovery messages [1], [2]. However, this simple scheme and similar techniques may not work as intended in duty cycled networks. In addition, operating on battery imposes a new criteria in designing protocols for sensor networks, especially, when they are used in critical infrastructures or in harsh conditions or hard-to-access fields that makes it impossible to replace or recharge them. In this regard, all protocols more or less require at least a reconsideration to reduce their operational energy demand.

Referring to the energy issue, neighborhood discovery has special importance as a mechanism providing the base information for other protocols such as routing. For example, calculating energy efficient routes or avoiding excessive retransmissions caused by interference, depend on detecting

This research was conducted, when Hamed Khanmirza was a visiting researcher in Chalmers University of Technology. suitable neighbors. This is why finding a suitable set of neighbors as quickly as possible often translates into energy efficiency [3], [4]. In addition, neighborhood discovery is a continuous process even in static sensor networks due to the wireless links dynamics which happens as a result of moving objects, interference with other networks or change in the weather condition [5]. As a result, sensors have to broadcast or exchange special discovery packets periodically to keep a uptodate set of neighbors. This continuous process adds an extra source of energy dissemination especially in LPLbased MAC protocols where sensors are mostly in sleep mode to conserve energy. Despite this importance few works have been done on designing an energy efficient neighbor discovery protocol comparing with available literature on routing or topology control protocols.

Low Power Listening (LPL) is a common MAC-Layer technique for reducing power consumption in wireless sensor networks [8]. By using a LPL MAC protocol, sensor nodes are switched on and off asynchronously or synchronously to reduce their radio hardware idle times. Such alternation is effective on decreasing the energy dissemination since it is shown that in small sensors, radio hardware is the major source of energy consumption [6].

Asynchronous LPL MACs are attractive because of their simplicity and cost of deployment for large-scale networks as they don't require synchronization or special pre-configuration. In asynchronous LPL MACs, sensor nodes usually wakeup periodically to perform a relatively short Clear Channel Assessment (CCA) to detect a probable channel activity. They remain active to receive the incoming packet information when they detect energy on the channel; otherwise, they go to sleep mode immediately. The length of this wake-up period in homogeneous networks is a network-wide parameter and is configured for every node at deployment time.

Although asynchronous scheme brings simplicity to MAC protocols, however, as sensors are not synchronized they don't have a clear clue about their neighbors' actual wake-up time. To address this problem, transmitters send a preamble to inform the next hop and keep it awake. Different protocols have distinct preamble types with various lengths. Among available protocols, X-MAC [8] and BoX-MAC [9] utilize a shorter preamble by applying a smart technique. They send short preambles repetitively with small gaps between successive 
transmissions to provide an opportunity for receiver to reply earlier. Most importantly, they embed the next packet or its header inside the preamble to eliminate overhearing problem. By this trick, they speed-up the actual communication as the receiver have the enough information about the transmitter and the intended target.

It is apparent that efficiency of traditional model of neighborhood discovery is questionable under asynchronous LPL MAC mechanisms. When a node broadcasts discovery beacons, simply, there is no guarantee that in that specific time all of its neighbors are awake. To ensure all neighbors reception, the broadcasting must be continued for a longer time that can be another source of energy waste. In higher density sensor networks flooding methods can also lead to a remarkably negative impact on throughput due to communication channel throttling.

In this paper, we study the possibility of using a completely passive, low-power and zero-overhead neighbor discovery method, specifically operating in conjunction with asynchronous LPL MACs. The key point behind the passive sensing strategy is to benefit from the information obtained from decoding of the packets during CCA checks. This implies absolutely no cost for sensor nodes, as periodic CCA checks and decoding of the received packets in case of energy detection inside the channel, are routine tasks that all nodes should perform when they wake-up. In addition, we argue that integrating neighbor discovery protocol to LPL MAC layer, not only simplifies the whole mechanism significantly, but also helps to smarter reduction of power consumption. The rest of the paper organized as follows. Next section gives a brief review on available solutions for low duty cycle MAC protocols. Section III presents our discovery method and discusses about its advantages. In section IV we develop a mathematical model for measuring performance of the new discovery method. In section $\mathrm{V}$ we evaluate our method by means of simulation and implementation in TinyOS. We also compare these results with the performance figures achieved by model. Finally, section VI concludes the paper and introduces our next steps to extend this approach.

\section{BACKGROUND AND RELATED WORK}

Power consumption is a major concern in designing communication protocols for sensor networks. Although some mechanisms emphasize on adjusting transmission power for power consumption reduction, researchers have found that the active radio is the main source of energy consumption. Authors in [6] give a simple example on how effective use of duty cycling can prolong the sensor life time from days up to several years. Due to this importance, various techniques and protocols emerged for MAC layer over the years which mostly focus on minimizing the radio active time to save more energy [7]-[16].

Low power MAC techniques usually are categorized into two broad classes of synchronous and asynchronous. Synchronous MAC layers utilize local or global schedules in order to wake-up nodes simultaneously for communication [11], [12]. As indicated in literature, synchronization have overheads and numerous deficiencies like scalability issue. To overcome these shortcomings, hybrid approaches that add some asynchronous functionalities into the pure synchronous protocols emerged [13], [14]. WiseMAC [13] works like Aloha and uses preamble sampling similar to LPL MACs. However, in WiseMAC transmitters are synchronized with each other through learning the next hop wake-up times. In contrast, SCPMAC [14] aligns the time of receive checks among nodes. The common drawback for synchronous MAC protocols is their relatively high degradation of performance against clock drifts or temperature shifts [10].

By introduction of Clear Channel Assessment (CCA) capability in some radio chips, Low Power Listening (LPL) protocols come into literature. CCA allows sensors check activity of the channel at a very lower power level without fully powering the radio. This helps not only to reduce the power usage but also removes the warm-up delay required for switching the radio on or go to deep-sleep state. B-MAC [7] was the first successful implementation of pure asynchronous low duty cycling mechanism. According to B-MAC, when a sender intends to transmit data, it should first transmit a long preamble with the length of network wake-up period. Upon hearing the preamble, all surrounding nodes stay awake to receive the subsequent transmissions. After elapsing a network period, the transmitter sends the actual data packet which also contains the information of the intended receiver. All receivers except the target node, which were awake so far go to sleep mode. In silent times, sensors wake up and assess the channel only for a short time. With this technique B-MAC completely eliminates the need for explicit synchronization.

The major drawback of B-MAC is its long, bit-stream preamble which results in overhearing problem and higher energy consumption. X-MAC [8] and BoX-MAC [9] suggest sending short preambles with gaps between successive preamble transmissions. Using gaps provide opportunity for early acknowledgment of the receiver and decrease preamble transmission time. Furthermore, they include the receiver id or the whole data packet inside the preamble to eliminate overhearing problem.

LPL-based MAC protocols are very common in current sensor networks because of their simplicity and low power operation beside other attractive characteristics like reliability, quick topology formation, and low maintenance cost [9]. This widespread use provokes designing more specialized protocols that can cooperate with LPL mechanism to save more energy.

Neighborhood discovery that is our focus in this paper, mostly is implemented in higher levels of network stack [6] and principally don't benefit from special radio features. Most of the suggested mechanisms utilize a discrete model of time called slots. Slots are supposed to be intervals of real time of sufficient length to permit communication. Each sensor either is awake or asleep in any given interval. Two nodes can discover each other if they both are awake simultaneously at the same slot. In [6] discovery methods are classified into 3 categories. First set of methods suggest exploiting randomness to choose the slot activity. Nodes randomly decide to transmit beacon, or listen to the channel to hear others beacon, or remain asleep at each slot. In this way, nodes probabilistically will find each other. The second set, recommend using special wake-up patterns that guarantee simultaneous wake-up at some point; for example, neighbor sensors should have prime sleep periods relative to each other. The last class of methods simply advise to remain active for certain successive number of slots 
to assure neighbor discovery. We refer the reader to [6] for detailed discussion on the mechanisms.

The main drawback of all of these mechanisms is the notion of time slot. Several practical issues are enumerated in [6] for determining duration of a slot. Such considerations cause the length of a time slot to be longer than expected. In addition, even in circumstances, despite simultaneous wakeup of sensors, mutual recognition may fail due to time slot's boundary incorrespondence. Such practical issues prevents further reduction of sensors wake-up state length and makes them less compatible with low duty cycle MAC protocols which are struggling to cut the duty cycle under $1 \%$. For the same reason, the class of methods trying to discover neighbors by widening the awake state duration may not be applicable in these networks.

Selecting specific time slot lengths distributedly to ensure coincidence of sensors' wake-up times (i.e. prime number), have also potential of turning into a repeated, rather complex process similar to the synchronization process in synchronized, or hybrid MAC protocols [17], [18], which is intentionally avoided by asynchronous protocols to stay simple and costeffective. We should also add scalability problem in large scale sensor networks to the previous remarks.

Another shortcoming subject to higher level neighborhood discovery protocols is their reliance on MAC layer for reliable packet delivery and taking care of collisions. Although this approach can decouple the protocol from the lower level considerations, but higher level protocols are not aware of broadcasting costs or packet transmission costs to a specific target. in turn may limit the amount of energy-saving.

\section{PASSIVE NeIGHBORHOOD Discovery Method}

Sensors have limited capabilities from various aspects like power, storage and computation; as a result, handling high volume of traffic, or performing a complex analysis on gathered data are kind of tasks that is never expected on a sensor. As an instance, in an event-driven sensor network, each sensor monitors and reports only noticeable changes in one or several environmental variables, like temperature, or patients vital signs. Apparently, in such scenarios, reports are transmitted using small packets and depending on application, traffic rate is considerably lower than what is usual in wireless ad-hoc networks. In addition, sudden bursts of traffic can be restricted in various points thanks to the data aggregation and data fusion techniques [19], [20]

This facts motivated us to investigate effectiveness of utilizing a passive sensing approach for neighborhood discovery. With passive sensing term, we refer to exploiting regular CCA checks as a unique source of information for the neighborhood discovery comparing with other techniques which actively take advantage of sending and receiving special discovery packets.

Passive neighborhood discovery has several advantages over active approaches. At first, this method does not consume additional energy for discovery purposes. Besides, there is no abstraction from the underlying MAC layer which can be a source of consuming extra energy. Moreover, passive sensing discovery can easily be integrated into existing LPL MAC protocols without noticeable overhead. Recent LPL MAC protocols usually are built around IEEE 802.15.4 standard [21] and transmitted packets include source and destination addresses in their header. Using B-MAC, sensors have to remain in active state until the next period to receive the packet. After receiving the full packet, all sensors can extract the required sender information from the header. X-MAC, however, needs a small change in preamble to be compatible with passive discovery. Preamble of X-MAC contains only the receiver id and all non-target nodes after decoding the preamble go to sleep state. To facilitate discovery in this phase before nodes go to sleep, sender id should be added to the preamble. But this change increases the length of preamble which may delay the starting of a communication. In contrast to X-MAC, BoX-MAC ${ }^{1}$ requires absolutely no change for passive neighbor discovery, since it sends the full packet as the preamble. BoX-MAC is the most minimal of the protocols and it is also the default MAC protocol for TinyOS 2.1 [22] with far better energy efficiency than X-MAC [9]. Due to its popularity and also simplicity of integrating the passive sensing into it, we selected BoX-MAC-2 as our default protocol in experiments.

As we will show in the next section, the proposed method is more sensitive to network activity than duty cycle length. This means that the passive discovery approach has better performance in dense networks or in networks having higher activities. In other scenarios, such as almost-silent networks or real-time applications, the passive approach may not be a suitable method to find neighbors, even though this method is flexible enough that can cooperate with available discovery methods. One can use passive discovery approach as the primary neighbor discovery method to conserve energy, but in case of failure, other supplementary methods can be activated.

\section{PERFORMANCE ANALYSIS}

In this section, we develop a mathematical model to measure the performance of passive neighborhood discovery algorithm. Then, in the next section we prove accuracy of the model through different experiments.

We assume a multi-hop homogeneous sensor network consisting of sensors having wake-up period of length $T$ and equipped with omnidirectional radio antennas. Sensors connect together with the classic unit disk graph (UDG) [23] connectivity model that means two nodes are called neighbors if they reside in each others transmission range. Transmission range of each node is simply a circle of radius $r$. Although, this perfect disk model seems very simplistic, however, it allows us to obtain essential formulation on dynamics of the network under various conditions and parameters.

In addition, we suppose an event-driven sensor network in which events inter-arrival time is exponentially distributed with the average rate of $\frac{1}{R}$ and they are independent of each other. Here, we consider only event-driven networks, since periodic networks may have better performance under adaptive approaches like WiseMAC [13]. As discussed, in asynchronous LPL MAC protocols transmitters have to send a preamble for duration of at most $T$ that can be modeled by a uniform distribution $U(0, T]$.

\footnotetext{
${ }^{1}$ Specifically BoX-MAC-2
} 


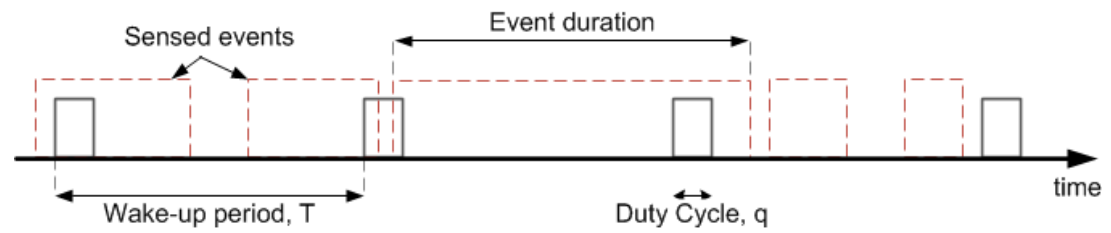

Fig. 1. This figure illustrates concept of duty cycle and events that can be sensed.

Let $u$ be a node under observation which will be called the passive node throughout this paper and have the duty cycle of length $q$ where $(q \ll T)$. The objective is to measure the Probability of Sense $\left(P_{o} S_{n}(k)\right)$ for network $N(R, T, q)$ at $k^{t h}$ cycle with presence of $n$ active transmitter in neighborhood of $u$. For conciseness we refer to $\operatorname{PoS}_{1}(1)$ by $P o S$.

\section{A. Probability of Sense}

We begin performance analysis by the following theorem:

Theorem 1: For a passive sensor node with duty cycle of length $q$ in a sensor network with wake-up period of $T$ and independent arrivals of events with mean rate of $\frac{1}{R}, P o S$ is obtained from the following equation:

$$
P o S=\left(1-e^{-\frac{q}{R}}\right)+\left(1-e^{-\frac{T-q}{R}}\right)\left(\frac{T+q}{2 T}\right)
$$

Proof: If we assume that the passive node can sense the activity of the channel instantly, PoS simply is the probability of the number of the transmissions fall within the sensible range. During each wake-up period, $u$ can sense two types of transmissions (see Fig.1):

1) Transmissions occur in the time interval $[0, q]$ which will definitely be sensed.

2) Transmissions occur in the time interval $[q, T]$ and stay active until the next duty cycle. In other words, if a transmission occur in time $t$ and stays for $s$ it will be sensed by $u$ if $t+s \geqslant T ; q<t<T$.

For the first period $k=1$ we can write:

$$
P o S=P_{\text {transmit }}(0, q)+P_{\text {transmit }}(q, T) \times P(s+t \geqslant T)
$$

$s$ is a uniform random variable between $(0, T]$ and $t$ is another uniformly distributed random variable in range $(q, T)$, then we have;

$$
\begin{aligned}
p(s+t \geqslant T)= & \int_{0}^{T} \int_{q}^{T} f_{s, t}(s, t) d s . d t= \\
& \int_{0}^{T} \int_{q}^{T} \frac{1}{(T-q)} \cdot \frac{1}{T} d s . d t=\frac{T+q}{2 T}
\end{aligned}
$$

On the other hand, events obey Poisson distribution with parameters $\lambda=\frac{1}{R}$ and $t=q$ :

$P_{\text {transmit }}(0, q)=1-P_{\text {no_transmit }}(0, q)=1-e^{-\frac{q}{R}}$

$P_{\text {transmit }}(q, T)$ could also be obtained similarly. Combining together, $P o S$ can be achieved by the following equation:

$$
P o S=\left(1-e^{-\frac{q}{R}}\right)+\left(1-e^{-\frac{T-q}{R}}\right)\left(\frac{T+q}{2 T}\right)
$$

Corollary 1: If there are $n$ transmitting neighbors around the passive sensor node, and assuming interference-free environment, probability of sense for the first period is $\mathrm{PoS}_{n}=$ $\left(1-e^{-n \frac{q}{R}}\right)+\left(1-e^{-n \frac{T-q}{R}}\right)\left(\frac{T+q}{2 T}\right)$.

Proof: We know that $n$ identically distributed Poisson variables can be combined in one Poisson random variable with mean rate of $n$ times of the original variables' mean rate. In an ideal condition without collision we can write:

$$
P o S_{n}=\left(1-e^{-n \frac{q}{R}}\right)+\left(1-e^{-n \frac{T-q}{R}}\right)\left(\frac{T+q}{2 T}\right)
$$

Not surprisingly, $\operatorname{Po}_{n}$ shows that the chance of sensing a transmission is higher when there are more active transmitters around the listening node. Hence, in the $k^{t h}$ period, we expect discovering more neighbors when there exist higher number of transmitting neighbors. But we should remember, since we assumed transmitters generate events completely independent of each other, the average and the worst case value of $P o S_{n}$ and $P o S$ are the same.

An important assumption in the analysis above is that there is no collisions between transmitting neighbors and they don't use back off timers to postpone the transmissions. In addition, it is presumed implicitly that parallel transmissions don't collide and consequently can be heard and sensed by the passive node. Despite this over simplistic model, in evaluation section, we will show that this simple model is enough to have overall insight on performance of passive sensing neighborhood discovery.

Theorem 2: Under the settings of Theorem 1 and constant network period, $P o S_{n}$ is bounded.

Proof: Intuitively, minimum value of the function occurs when $q \rightarrow 0$, because by increasing $q$ chance of sensing more transmissions raises. Thereby, we can find the lower bound by limiting $q$ toward 0 , while keeping $T$ constant: $\lim _{q \rightarrow 0} \operatorname{PoS}_{n}=\frac{1}{2}\left(1-e^{-n \frac{T}{R}}\right)$.

On the other hand, maximum probability of sense is achieved when the passive node is always on which means: $\lim _{q \rightarrow T} P_{o} S_{n} \approx\left(1-e^{-n \frac{T}{R}}\right)$.

$$
\frac{1}{2}\left(1-e^{-n \frac{T}{R}}\right) \leqslant P o S_{n} \leqslant\left(1-e^{-n \frac{T}{R}}\right)
$$

Theorem 2 reveals a very interesting point about the relation of duty cycle length and probability of sense. According to equation (2), even with a fairly small duty cycle, passive sensing node can capture enough information about its neighbors and there is always a definite, positive gain of information which is more than $50 \%$ of all possible transmissions may 
occur in a period. This is due the fact that value of $T$ not only controls the sensing interval, but also controls the extent to which transmitters should keep the channel active before the neighbor get informed. Figure 2a illustrates the bounds with various duty cycle lengths in presence of one transmitter. In this figure, duty cycle lengths are abnormally higher than what is used in actual deployments which is around $1 \%$ of the wakeup period, but here they are used for representation purposes only.

Corollary 2: Increasing wake-up period length, increases the $P o S_{n}$.

Proof: In asynchronous LPL MAC protocols duty cycle normally is less than $1 \%$ of wake-up period then we can assume $T \gg q$. With this assumption fraction $\frac{T+q}{2 T}$ in equation (3) approaches to $\frac{1}{2}$. Knowing that expression $\left(1-e^{-n \frac{T-q}{R}}\right)$ is an increasing function of $T$, we can conclude that equation (3) is also an increasing function of $T$.

This corollary is important from this perspective that even in low rate monitoring applications, there exists an acceptable chance to get information from the surrounding network which is illustrated in Fig.2b. This figure inspects the variation of $P o S$ from the perspective of wake-up period length and small duty cycles $(1 \%-20 \%)$. In this figure, higher sensitivity of $P o S$ to $T$ is more clear. While $P o S$ increase exponentially with $T$, increasing length of duty cycle have no noticeable influence on it.

Corollary 3: Discovery process slows down for slow sensor applications

Proof: This corollary is a natural result of passive sensing approach which relies on the sensors' activity in order to discover neighbors. If the changes of the monitored variables are rare, sensors will be quiet at most of the times and discovery process will be slow. Taking the derivative of equation (3) with respect to $R$ results in function $P_{o} S^{\prime} \approx\left(e^{q}+\right.$ $\left.\frac{1}{2} e^{(T-q)}\right) \cdot e^{-\frac{1}{R}} \cdot \frac{1}{R^{2}}$ which is monotonically decreasing function of $R$ and confirms the above explanation, mathematically.

As Fig.2c indicates, discovery speed experience an exponential decrease in connection with application mean rate. This means that if in a scenario, $R$ is doubled, to achieve the same $P o S$, nodes have to double the $T$. In Fig.2d, a sensor has $P o S$ of around 0.05 with $R=60$, while the same $P o S$ with $R=180$ is achieved with wake-up period length of 18 .

\section{B. Neighbor Discovery Speed}

Up to now, we calculated and analyzed the probability of sense for one wake-up period. In this section, we present analysis on neighborhood discovery speed. We begin by measuring the time that is required for sensing the first neighbor. Discovering the first neighbor is important in the sense that nodes can communicate with outer world with having only one neighbor, although this communication may not be optimized with respect to energy usage. Hence, it is crucial to know that how much time a passive node should wait to find at least one hop around itself.

Based on assumptions, discovery on each period is independent of the other periods. Then, the probability of sense of a neighbor in each period is like a Bernoulli trial with the

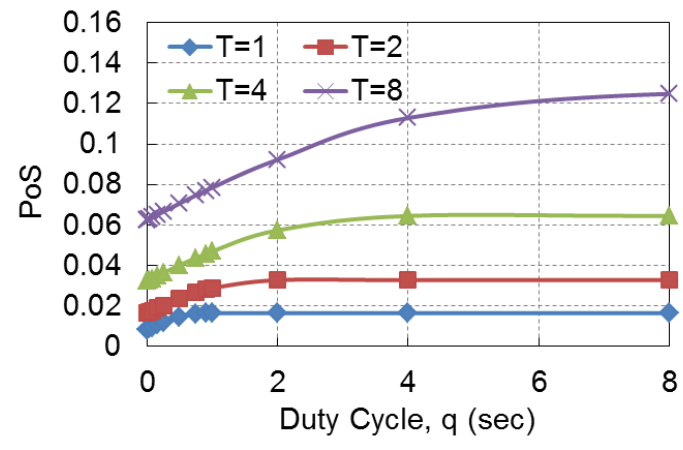

(a) $P o S$ against various duty cycles (q) $(\mathrm{n}=1, \mathrm{R}=60 \mathrm{~s})$

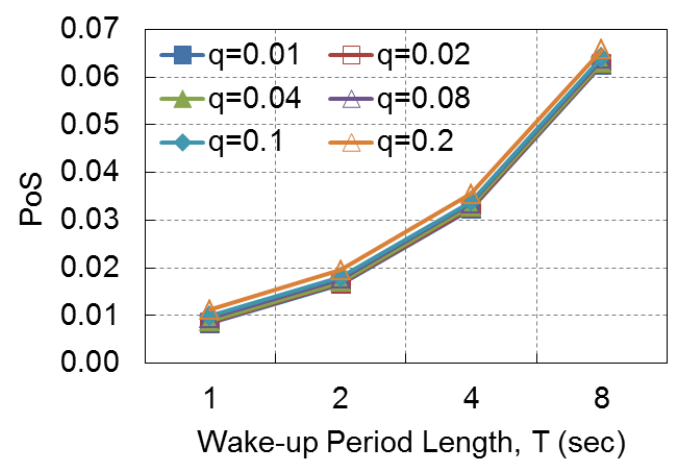

(b) $P o S$ against various wake-up period lengths $(\mathrm{n}=1, \mathrm{R}=60 \mathrm{~s})$

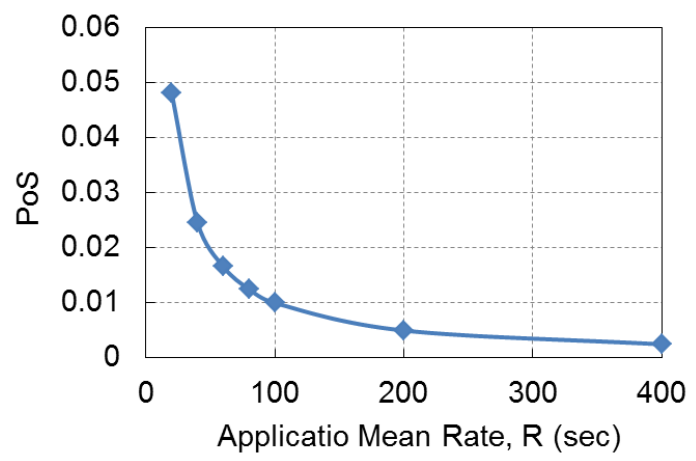

(c) $\operatorname{PoS}$ variation with different application mean rates $(\mathrm{n}=1$, $\mathrm{T}=2 \mathrm{~s}, \mathrm{q}=11 \mathrm{~ms}$ )

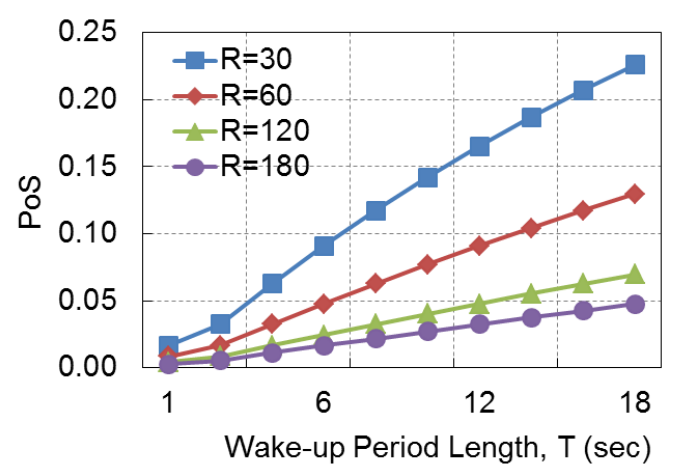

(d) $P o S$ varition with different wake-up lengths ( $\mathrm{n}=1, \mathrm{q}=11 \mathrm{~ms}$ )

Fig. 2. Dynamics of Probability of Sense

success probability of $\mathrm{PoS}_{n}$. Based on this model, probability 


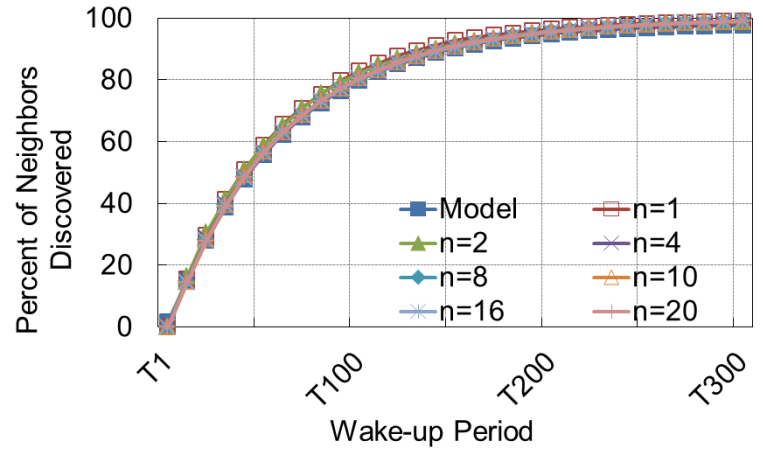

(a) Percent of discovered neighbors

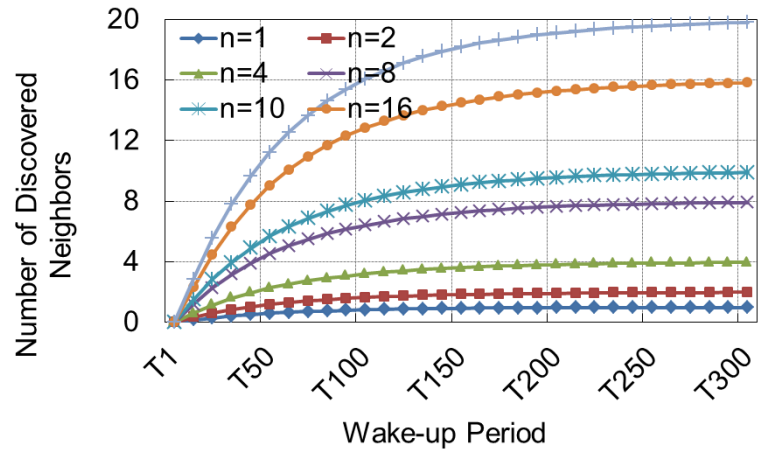

(b) Number of discovered neighbors

Fig. 3. Neighbor Discovery speed with different number of neighbors in various wake-up periods having duty cycle of $0.5 \%(\mathrm{~T}=2 \mathrm{~s}, \mathrm{R}=60 \mathrm{~s}, \mathrm{q}=11 \mathrm{~ms})$

of success in $k^{t h}$ round corresponds to variable $P_{o} S_{n}(k)$ that is a geometric random variable:

$$
\operatorname{PoS}_{n}(k)=\operatorname{PoS}_{n} \cdot\left(1-\operatorname{PoS}_{n}\right)^{k}
$$

The next useful metric for getting an idea on the speed of neighborhood discovery is to find the probability of sensing all neighbors in each period, $P o S_{a l l}(k)$. For one transmitter, probability of sensing the transmitter before the $k^{\text {th }}$ period is the reverse of the situation where no transmission has been sensed before that period: $1-\left(1-P_{o} S\right)^{k}$. Extending this formula for $n$ independent transmitters get the following expression:

$$
\operatorname{PoS}_{\text {all }}(k)=\left(1-(1-P o S)^{k}\right)^{n}
$$

\section{Performance Evaluation}

In previous section, we developed an analytical model for discovery performance of our new method. In this section, we evaluate performance of the passive neighborhood discovery method along with its model using both simulation and implementation. At first, we verify the theoretical correctness of model by simulation without considering usual practical issues. Then, we implement the discovery method in TinsOS 2.1 to check how this method performs in presence of interference and other real world parameters.

\section{A. Simulation}

Simulations conducted with different number of transmitters sending events toward a sink node which is located by one hop from all of them. Another sensor in network, plays the role of passive sensing node and does not send or receive any packet. We place this node in a way that it can snoop all transmissions. To keep the simulation as simple as possible, we didn't include influence of interference and back off timers in simulated model. However, passive node can just sense a transmission if it is the only active transmission in the network and ignores any parallel transmissions to simulate jamming and packet corruption.

Events generated in the network by the rate of one packet per minute $(R=60)$ with the length selected by random in the $(0, T]$ range.To ensure the stability of the results, each simulation is repeated for 1000 times and final values averaged over all runs.

Fig.3a shows the average percent of discovered neighbors in the $k^{\text {th }}$ period. Since transmitters work independently the average percent of discovered neighbors is equal for all configurations. The important point here is the correspondence between the simulation and the model curves despite the fact that we removed the complexity of modeling collisions from our mathematical model. The next figure shows the same statistics with emphasize on the actual number of discovered neighbors. Based on this figure, the chance of sensing more neighbors is higher by average when there are more neighbors around the passive node which confirms the correctness of equation (3). Under the settings of the simulation, transmissions have mean rate of 1 packet per minute, and passive sense node was able to discover $50 \%$ of its neighbors in around 80 seconds. This is an interesting result because it denotes that in a medium density sensor network (neighbor count 4-8) each node with high probability will found half of its neighbors in time less than $2 R$.

Finally, we measured the speed of sensing all available neighbors that is shown in Fig.4. In some cases it takes too long time to discover all neighbors; for instance, in a medium density network, it is possible for a node not to find all of its neighbors after more than 4.R. Apart from the results, once again there exists a close correspondence between model and simulation results.

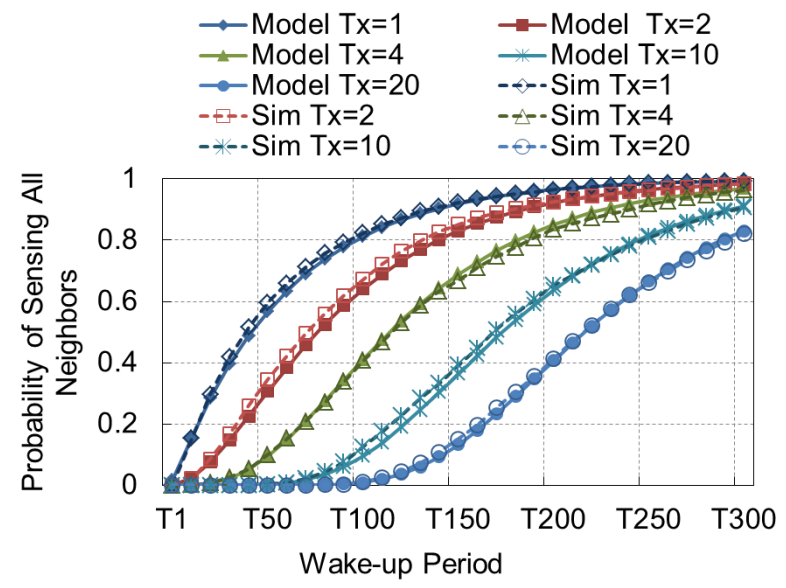

Fig. 4. Probability of sensing all neighbors in different periods with different number of neighbors 


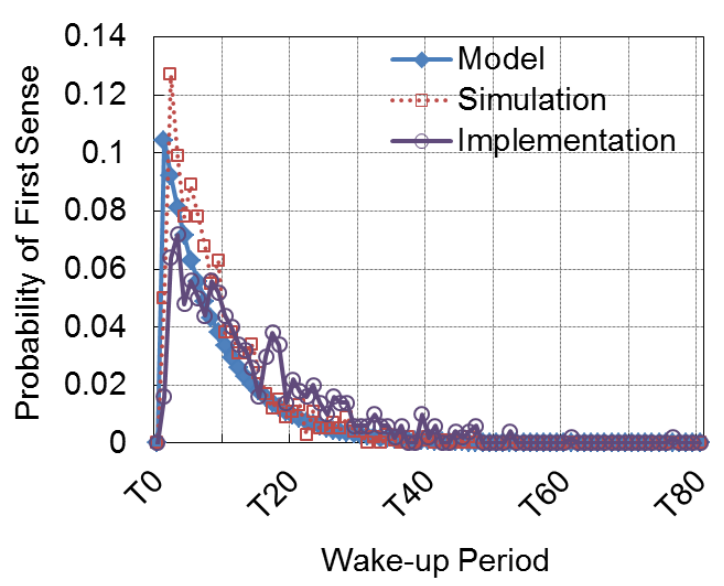

(a) Probability of first neighbor sense in various periods $(n=8)$

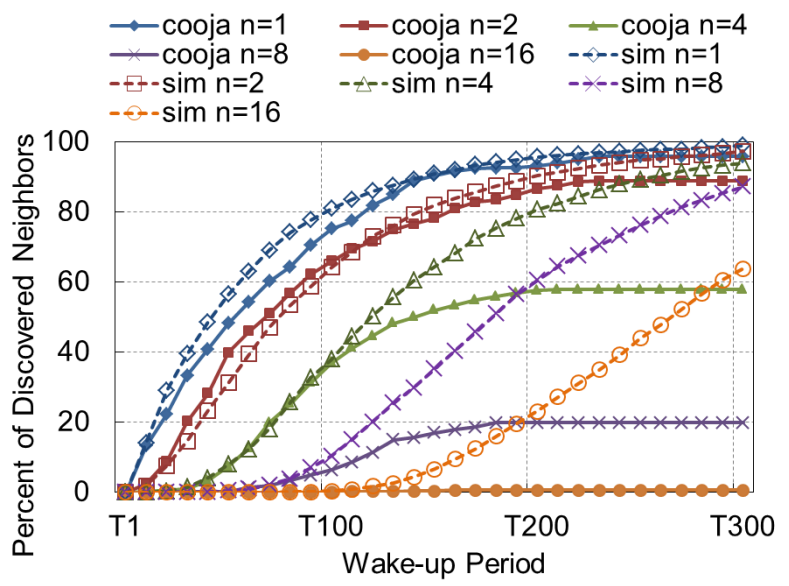

(b) Comparison of probability for sensing all of neighbors in Cooja and simulation

Fig. 5. First sense and all neighbors discovery speed $(R=60 s, T=2 s, q=11 \mathrm{~ms})$

\section{B. Implementation}

In order to verify the simulation results and also inspect interference and back off timers influence on $\mathrm{PoS}$, we implemented the passive sensing mechanism in TinyOS on TelosB [24] motes which are equipped with Chipcon CC2420 [25] radios. Several LPL MAC protocols especially BoX-MAC-2 are available out-of-the-box in TinyOS distribution for these radio chips. Experiments were done on Cooja simultaor [27] which is a cycle accurate network simulator developed for Contiki Operating System [26] and allows emulation of motes at hardware level.

To ensure adequate level of interference, sensor nodes arranged in a circular topology with one passive sensing node in the middle. Each sensor transmits data to the sensor having the next node id. Using this construction sensors have at least 4 other sensors in their transmission range. Passive sensor can snoop all transmissions in the region. We repeat the similar scenarios in the previous section and each scenario is repeated for 500 times to ensure stability in results.

Fig.5a presents the probability distribution of sensing the first neighbor in various wake-up periods for a configuration with neighborhood size of 8 . Passive sensor, in both simulation

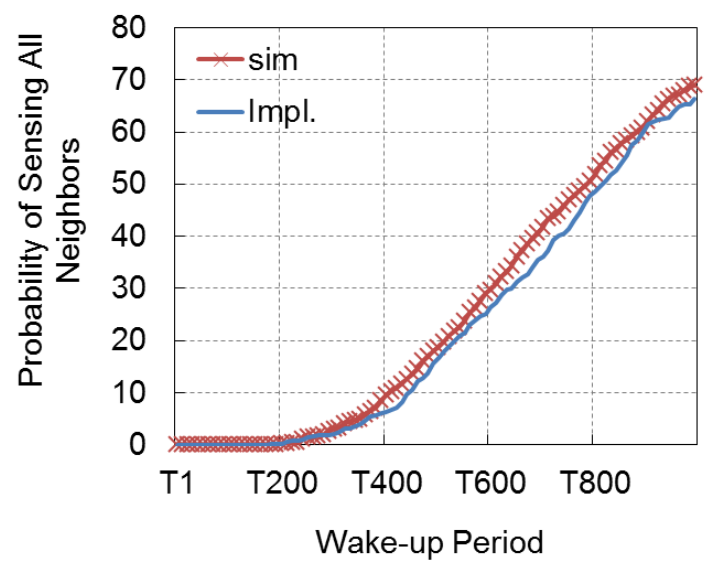

Fig. 6. Probability of sensing all neighbors for neighborhood of size 8 ( $n=8$, $\mathrm{T}=2 \mathrm{~s}, \mathrm{q}=11 \mathrm{~ms}, \mathrm{R}=240 \mathrm{~s}$ )

and implementation tests, finds its first neighbor in times less than $R$ with high probability and closely follows the model. As discussed before, finding the first neighbor is very crucial to begin the communication. Despite the presence of relatively high interference the probability of first neighbor sense is not affected. Due to similarity of the results for average discovery speed to the previous section, we don't repeat them here.

In contrast to other metrics, Fig.5b expresses a relatively large deviation on performance of discovering all neighbors between simulation and implementation. When number of the adjacent sensors goes beyond 3, the chance of detecting new transmitters remarkably decreases to the degree that in case of having 16 neighbors, there is almost no hope to find all neighbors. In scenario with 4 neighbors, passive node has only $50 \%$ chance, and for 8 neighbors this possibility falls to less than $20 \%$. From this numbers, we can expect that some of the neighbors will never be detected by the neighbor discovery procedure.

With further investigating the problem, we found that the main cause of the poor performance relates to the network performance. Because of high collision and relatively fast rate of reports, throughput of these networks degraded to such a low level that only little portion of packets could be sent over the network. In fact, with the condition of this experiment, a few valid transmissions occur in neighborhood.

To validate this claim, we reduced the mean rate of reports by 4 times without changing the topology. The new results, displayed in Fig.6, show an excellent correspondence between simulation and real application results. By this experiment, we can conclude that as long as the network operate in a good condition and is not throttled by other factors, passive sensing neighbor discovery will run close to what model and simulation anticipate.

\section{CONCLUSION AND FUture WORKS}

In this paper, we provided a base analysis on possibility of using passive sensing approach for neighborhood discovery to mitigate power consumption in sensor networks. We developed a mathematical model for measuring performance of our proposed method. The model at first is verified by 
simulation and then both model and simulation results are verified through implementation in TinyOS. Passive sensing neighborhood discovery adds absolutely no extra overhead on network protocol stack and only use the information achieved by routine CCA checks available in LPL MAC protocols. Moreover, by integrating into the MAC layer it removes some the energy costs incurred by high level discovery protocols.

Our analysis and also experiments showed that the passive sensing discovery is a reasonably efficient approach for discovering neighbors as long as no real-time requirements is demanded by the application. Currently, we are working on enhancing the discovery process with other mechanisms such as energy-efficient exchanging of the neighbor set between adjacent nodes to speed-up the whole discovery process and make it fault-tolerant.

\section{REFERENCES}

[1] RFC 4861, Neighbor Discovery for IP version 6 (IPv6), T. Narten et al. (September 2007)

[2] 6lowpan Working Group. http://datatracker.ietf.org/wg/6lowpan/charter

[3] S. Vasudevan, On neighbor discovery in wireless networks with directional antennas. INFOCOM 2005. 24th , 2005.

[4] A. Kandhalu, K. Lakshmanan, and R. Rajkumar, Neighbor discovery in mobile sensor networks, 2010.

[5] M. Kohvakka, J. Suhonen, M. Kuorilehto, V. Kaseva, M.Haännikäinen, and T. D. Hämäläinen, Energy-efficient neighbor discovery protocol for mobile wireless sensor networks, Ad Hoc Networks, vol. 7, no. 1, pp. 2441, 2009.

[6] V. Galluzzi and T. Herman, Survey: Discovery in Wireless Sensor Networks, International Journal of Distributed Sensor Networks, vol. 2012, pp. 112, 2012.

[7] Polastre, Joseph, Jason Hill, and David Culler. Versatile low power media access for wireless sensor networks. Proceedings of the 2nd international conference on Embedded networked sensor systems. ACM, 2004.

[8] Buettner, Michael, et al. X-MAC: a short preamble MAC protocol for duty-cycled wireless sensor networks. Proceedings of the 4th international conference on Embedded networked sensor systems. ACM, 2006.

[9] Moss, David, and Philip Levis. Box-macs: Exploiting physical and link layer boundaries in low-power networking. Technical Report SING-0800, Stanford University, 2008.

[10] J. Arfvidsson, E. Park, and P. Levis. Lowering radio duty cycle through temper- ature compensated timing. Proceedings of the 4th international conference on Embedded networked sensor systems, pages 409410, 2006.

[11] W. Ye, J. Heidemann, and D. Estrin. An energy-efcient MAC protocol for wireless sensor networks (SMAC). Twenty-First Annual Joint Conference of the IEEE Computer and Communications Societies (INFOCOM), 3:15671576, June 2002.

[12] T. van Dam and K. Langendoen. An adaptive energy-efcient MAC protocol for wireless sensor networks. Proceedings of the 1st international conference on Embedded networked sensor systems, pages 171180, 2003.

[13] A. El-Hoiydi and J. Decotignie. WiseMAC: an ultra low power MAC protocol for the downlink of infrastructure wireless sensor networks. Proceedings. ISCC 2004. Ninth International Symposium on Computers and Communications, 1:244251, June/July 2004.

[14] W. Ye, F. Silva, and J. Heidemann, Ultra-low duty cycle MAC with scheduled channel polling, Proceedings of the 4th international conference on Embedded networked sensor systems - SenSys 06, p. 321, 2006.

[15] W. Ye, J. Heidemann, and D. Estrin. An energy-efficient mac protocol for wireless sensor networks. In In Proceedings of the 21st International Annual Joint Conference of the IEEE Computer and Communications Societies (INFOCOM 2002), June 2002.

[16] A. El-Hoiydi. Aloha with preamble sampling for sporadic traffic in ad hoc wireless sensor networks. In Proceedings of IEEE International Conference on Communications, Apr. 2002
[17] T.Herman,S.Pemmaraju,L.Pilard,andM.Mjelde,Temporal partition in sensor networks, in Stabilization, Safety, and Security of Distributed Systems (SSS 07), vol. 4838 of Springer Lecture Notes in Computer Science, 2007.

[18] A. Kandhalu, K. Lakshmanan, and R. Rajkumar, U-connect: a lowlatency energy-efficient asynchronous neighbor discovery protocol, in Proceedings of the 9th International Conference on Information Processing in Sensor Networks (IPSN 10), pp. 350361, April 2010.

[19] Krishnamachari, L., Deborah Estrin, and Stephen Wicker. The impact of data aggregation in wireless sensor networks. Distributed Computing Systems Workshops, 2002. Proceedings. 22nd International Conference on. IEEE, 2002.

[20] Luo, Hong, et al. Adaptive data fusion for energy efficient routing in wireless sensor networks. Computers, IEEE Transactions on 55.10 (2006): 1286-1299.

[21] Wireless medium access control (mac) and physical layer (phy) specifications for low-rate wireless personal area networks (lr-wpans). IEEE Standard 15.4, 2003.

[22] W. Zeng, J. Li, and A. Arora, Comparative Factor Analysis and Experimental Validation of Low Power MAC Performance, cse.ohiostate.edu, no. ii, 2011.

[23] S. Schmid and R. Wattenhofer, Algorithmic models for sensor networks, Proceedings 20th IEEE International Parallel \& Distributed Processing Symposium, p. 11 pp., 2006.

[24] Moteiv telosb motes. http://www.moteiv.com.

[25] Chipcon cc 2500 radios. http://www.chipcon.com.

[26] Contiki Operating System, http://www.contiki-os.org.

[27] Cooja Network Simulator: http://www.contiki-os.org/start.html. 